\title{
Bloch-phonon coupling and tunneling-induced coherent phonon excitation in semiconductor superlattices
}

\author{
M. Först* and H. Kurz \\ Institut für Halbleitertechnik, Rheinisch-Westfälische Technische Hochschule Aachen, D-52074 Aachen, Germany \\ T. Dekorsy \\ Institut für Ionenstrahlphysik und Materialforschung, Forschungszentrum Rossendorf, D-01314 Dresden, Germany \\ R. P. Leavitt \\ U.S. Army Research Laboratory, 2800 Powder Mill Road, Adelphi, Maryland 20783
}

\begin{abstract}
The coupling of Bloch oscillations to longitudinal optical phonons is investigated in a narrow-well $\mathrm{In}_{0.53} \mathrm{Ga}_{0.47} \mathrm{As} / \mathrm{In}_{0.52} \mathrm{Al}_{0.48} \mathrm{As}$ superlattice. A strong increase of coherent phonon amplitudes is observed when the Bloch oscillations are subsequently tuned into resonance with different optical phonon modes. The rapid dephasing of the Bloch oscillations due to field induced tunneling from the weakly bound miniband into above-barrier continuum states leads to an additional and new excitation process of coherent phonons in superlattices at high electric fields. Polarization dependent measurements confirm their generation via Coulomb interaction excluding any contribution through impulsively stimulated Raman scattering.
\end{abstract}

Even ten years after their first experimental observation, Bloch oscillations (BO's) of electronic wave packets, are still an intriguing phenomena in solid state physics. BO's have been intensively investigated from both an experimental and a theoretical point of view (for a review, see, e.g., Ref. 1). In general, BO are described as the spatial oscillation of carriers in a periodic potential subject to a static electric field., ${ }^{2,3}$ Their first experimental observation was accomplished in biased semiconductor superlattices. ${ }^{4,5}$ Here, the coherent superposition of electronic Wannier-Stark (WS) states by ultrashort laser pulses leads to the formation of an electronic wave packet oscillating with a frequency $\nu_{\mathrm{BO}}=e F d / h$. This frequency is determined by the externally applied electric field $F$, the superlattice period $d$, the electron charge $e$, and Planck's constant $h$.

Zener tunneling of charge carriers was initially investigated from the valence to the conduction band in bulk semiconductors at high electric fields. ${ }^{3}$ In semiconductor superlattices, field-induced tunneling was observed between different below-barrier electronic minibands ${ }^{6}$ and from below-barrier minibands to the above-barrier continuum states. ${ }^{7}$ In addition to these transport processes, the dynamics of BO were studied in shallow superlattices, demonstrating a rapid dephasing due to Zener tunneling into the abovebarrier continuum. ${ }^{8}$

In superlattices with a sufficiently large miniband width, BO can be tuned into resonance with longitudinal optical (LO) phonons giving rise to an enhanced interaction between these two excitations. A rapid dephasing of the electronic coherence due to resonant scattering processes between adjacent WS states has been predicted. ${ }^{9}$ On the other hand, the oscillating electronic wave packets can act as a resonant driving force for polar lattice vibrations via their associated longitudinal polarization. ${ }^{10}$ The latter effect of Bloch-phonon coupling was demonstrated experimentally for the case of the GaAs LO phonon in $\mathrm{GaAs} / \mathrm{Al}_{0.3} \mathrm{Ga}_{0.7} \mathrm{As}$ superlattices. ${ }^{11}$
The coupling of $\mathrm{BO}$ to the AlAs-like LO phonon related to the $\mathrm{Al}_{0.3} \mathrm{Ga}_{0.7} \mathrm{As}$ barriers, however, was not observed in these experiments.

Principally, ternary semiconductor layers exhibit a variety of LO phonon modes. Therefore, specific details of the coupling between BO and optical phonons of different frequencies can be investigated in these materials in the time domain. In addition, ternary superlattices with narrow wells and a shallow bound first electronic miniband open the way to study Bloch-phonon coupling in the presence of Zener tunneling at high electric fields.

Here, we present an experimental study of coupled Blochphonon oscillations in a narrow-well $\operatorname{In}_{0.53} \mathrm{Ga}_{0.47} \mathrm{As} /$ $\mathrm{In}_{0.52} \mathrm{Al}_{0.48} \mathrm{As}$ superlattice that consists of 100 periods of 8.4 $\AA$ thick $\operatorname{In}_{0.53} \mathrm{Ga}_{0.47} \mathrm{As}$ wells and $51.6 \AA$ thick $\operatorname{In}_{0.52} \mathrm{Al}_{0.48} \mathrm{As}$ barriers grown lattice-matched to an (001) InP substrate. These parameters result in a $60 \mathrm{meV}$ wide first electronic miniband that is shallow bound $20 \mathrm{meV}$ (upper edge) below the barriers of the conduction band while no higher bound electronic minibands exist. The superlattice is embedded in the intrinsic region of a $p-i-n$ diode in order to allow a variation of the electric field along its growth direction.

The optical phonons of the ternary well and barrier materials exhibit a typical two-mode behavior. The zero wave vector LO phonon frequencies of the unstrained bulk compounds are 7.1 THz (InAs-like) and 8.0 THz (GaAs-like) for $\mathrm{In}_{0.53} \mathrm{Ga}_{0.47} \mathrm{As}$, and 7.1 THz (InAs-like) and $11.0 \mathrm{THz}$ (AlAslike) for $\operatorname{In}_{0.52} \mathrm{Al}_{0.48} \mathrm{As} .{ }^{12,13}$ Thus, the electronic miniband width of $60 \mathrm{meV}$ (equivalent to $14.5 \mathrm{THz}$ in the frequency range) enables the coherent optical excitation of $\mathrm{BO}$ tunable across the LO-phonon frequencies of both, the superlattice well and barrier material. Note that the LO phonon branches of the InAs-like modes of bulk $\operatorname{In}_{0.53} \mathrm{Ga}_{0.47} \mathrm{As}$ and bulk $\mathrm{In}_{0.52} \mathrm{Al}_{0.48} \mathrm{As}$ overlap in the first Brillouin zone. Thus, they form delocalized optical phonon modes along the superlat- 


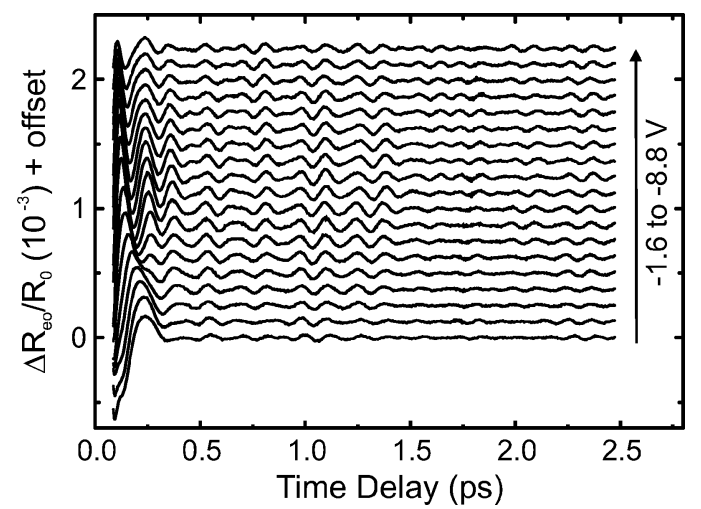

FIG. 1. Numerically extracted oscillations of the REOS signals detected in the voltage range between -1.6 and $-8.8 \mathrm{~V}$, i.e., in the Wannier-Stark regime of the superlattice. For clarity, voltage steps of $-0.4 \mathrm{~V}$ are shown here.

tice growth direction. In contrast, the frequencies of the GaAs-like LO phonon branch of $\operatorname{In}_{0.53} \mathrm{Ga}_{0.47} \mathrm{As}$ and the AlAs-like LO phonon branch of $\operatorname{In}_{0.52} \mathrm{Al}_{0.48} \mathrm{As}$ are strictly separated in the first Brillouin zone. Consequently, they remain confined in the wells and barriers, respectively.

The experiments were carried out in a femtosecond timeresolved electrooptic detection scheme, referred to as REOS (reflective electrooptic sampling). ${ }^{11,14}$ Here, polarization dynamics arising from optically excited coherent electron and lattice dynamics are monitored by anisotropic changes of the sample reflectivity due to the linear electrooptic effect. The laser pulses were derived from a Ti:sapphire oscillator tuned to a photon energy of $1.39 \mathrm{eV}$ with a spectral bandwidth of $50 \mathrm{meV}$ and a pulse duration of $45 \mathrm{fs}$. At a sample temperature of $10 \mathrm{~K}$, these laser parameters enable the excitation of BO by the coherent superposition of electronic WS states, i.e., $\mathrm{WS}(0)$ and $\mathrm{WS}(-1)$, over a wide voltage range.

REOS measurements were performed at a sample temperature of $10 \mathrm{~K}$ in the voltage range between $-1.6 \mathrm{~V}$ and $-8.8 \mathrm{~V}$, i.e., within the Wannier-Stark regime of the superlattice. The optical excitation density was kept low at a value of approximately $2 \times 10^{9}$ electron-hole pairs per well and $\mathrm{cm}^{2}$ in order to reduce carrier-carrier scattering and quasistatic screening of the applied electric field under high repetition rate excitation. The REOS signals typically consist of an initial contribution at zero time delay due to electrons and holes optically excited in a polarized state (not shown, see, e.g., Ref. 14). At later time delays, the signal traces exhibit oscillatory modulations that are induced by coherent electron and lattice polarizations along the superlattice growth direction. Extracted oscillations are depicted in Fig. 1 in voltage steps of $-0.4 \mathrm{~V}$. They are composed of two contributions that can be distinguished by their voltage dependence. The first component for time delays below $400 \mathrm{fs}$ is characterized by a frequency increase and a concomitant amplitude decrease with increasing reverse bias. The second component consists of a long-living frequency beat on a picosecond time scale that shows no discernable frequency changes but a remarkable amplitude increase if the applied voltage is varied.

For an unambiguous interpretation of the coherent polarization dynamics in the superlattice, the time-resolved signal

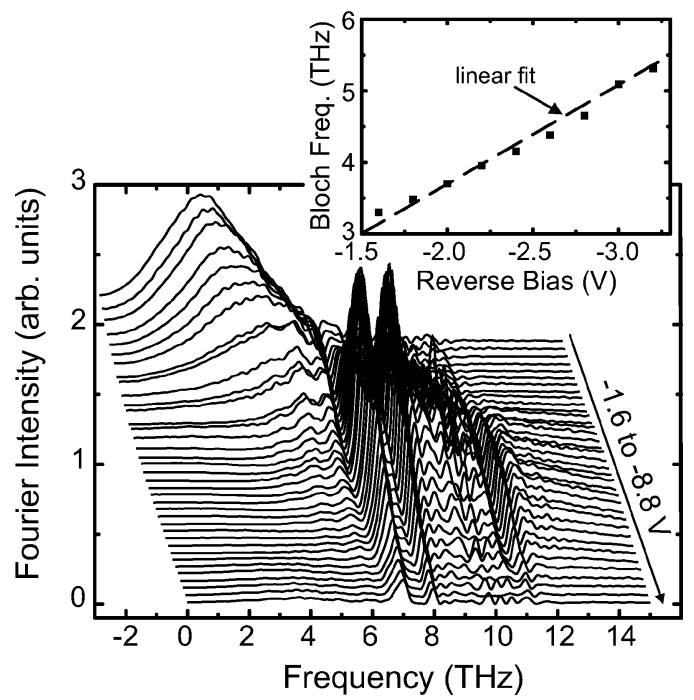

FIG. 2. Fourier transforms of the time-resolved REOS signals shown in Fig. 1 in voltage steps of $-0.2 \mathrm{~V}$. The spectra are shifted both vertically and horizontally. The inset shows the frequency of the $\mathrm{BO}$ in the low voltage range (see text).

modulations are Fourier transformed. The spectra obtained numerically are shown in Fig. 2 in voltage steps of $-0.2 \mathrm{~V}$. In the low-voltage range, the Fourier transforms are dominated by spectrally broad peaks arising from BO. As expected, their frequency increases linearly with increasing electric field applied to the superlattice. Concomitantly, the amplitude of the $\mathrm{BO}$ decreases. The peak positions of these contributions are determined for voltages up to $-3.2 \mathrm{~V}$ by Gaussian shaped numerical fits. ${ }^{15}$ The BO frequencies are shown as a function of the reverse bias in the inset of Fig. 2 . They depend linearly on the applied voltage with a slope of $-1.4 \mathrm{THz} / \mathrm{V}$ as obtained by a linear fit. This value is in good agreement with a theoretically expected slope of $-1.45 \mathrm{THz} / \mathrm{V}$ calculated from the potential period and the length of the intrinsic region of the $p-i-n$ diode thus corroborating the optical excitation of $\mathrm{BO}$.

The BO dephasing rate can be determined from the spectral linewidth in the Fourier transforms. Even below the lowest phonon frequency, we observe an increase of the dephasing rate as a function of the applied electric field. The spectral linewidths of the Bloch peaks increase from $2.9 \mathrm{THz}$ at a reverse bias of $-1.6 \mathrm{~V}$ to a value of $4.1 \mathrm{THz}$ at $-3.2 \mathrm{~V}$, corresponding to a decrease of the dephasing times from 350 to $250 \mathrm{fs}$. The increased dephasing even below any phonon frequency is in contrast to previous experiments in GaAs/ $/ \mathrm{Al}_{0.3} \mathrm{Ga}_{0.7} \mathrm{As}$ superlattices with a deeply bound first electronic miniband (see, e.g., Ref. 14). In the superlattice investigated here, the energy position of the electronic miniband $20 \mathrm{meV}$ below the barrier energy enables tunneling processes of the optically excited electrons into above-barrier continuum states. As a result, the electronic coherence is destroyed with an increased dephasing rate at higher electric fields. ${ }^{8,16}$ In addition, tunneling processes result in a fast change of the longitudinal polarization in the superlattice that can launch coherent optical phonons if the tunneling rate is higher than the phonon frequency (for a review on coher- 


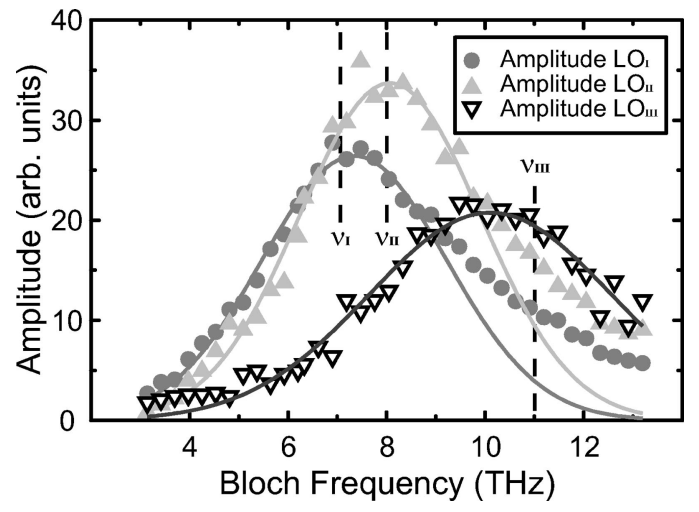

FIG. 3. Amplitudes of coherent LO phonons (see text) versus the BO frequency. The solid lines are Gaussian fits to the lowfrequency edge, dashed lines indicate the LO phonon frequencies.

ent phonon generation via electric field screening, see, e.g., Ref. 17).

Towards the high-voltage range, three sharp peaks at frequencies of (I) 7.1, (II) 8.0, and (III) 11.0 THz mainly contribute to the Fourier transforms shown in Fig. 2. These peaks result from the long-living beat in time domain and their frequencies correspond to the distinct optical phonon modes of the superlattice as introduced above. The amplitudes of the $\mathrm{LO}_{\mathrm{I}}$ and $\mathrm{LO}_{\mathrm{II}}$ phonons are considerably enhanced in the voltage range around $-5.0 \mathrm{~V}$, while the amplitude maximum of the $\mathrm{LO}_{\text {III }}$ phonon is less developed and shifted to a higher voltage. The voltage dependent increase of the coherent phonon amplitudes is ascribed to their coupling to the optically excited BO that leads to an efficient coherent phonon excitation under resonance conditions.

To confirm this picture, the coherent LO phonon amplitudes are plotted versus the BO frequency in Fig. 3. For this purpose, the voltage dependent $\mathrm{BO}$ frequencies (shown in Fig. 2) are linearly extrapolated to beyond the phonon frequencies. The coherent phonon amplitudes are determined from Fourier transforms for time delays larger than $300 \mathrm{fs}$, thus omitting contributions of the rapidly dephasing BO. Their relative amplitudes are corrected for the duration of the detecting laser pulses that leads to a decrease of the oscillation amplitudes at higher phonon frequencies. The phonon amplitudes show pronounced maxima at certain phonon eigenfrequencies. Their distinct peak positions are determined from Gaussian shaped numerical fits to the rising edge of the data, additionally shown in Fig. 3. The $\mathrm{LO}_{\mathrm{I}}$ and $\mathrm{LO}_{\mathrm{II}}$ phonons reach their amplitude maxima at $\mathrm{BO}$ frequencies of 7.3 and $8.0 \mathrm{THz}$, respectively. These values coincide well with the corresponding phonon frequencies of 7.1 and 8.0 $\mathrm{THz}$, thus demonstrating a selective coupling between the optically excited BO and the delocalized InAs-like LO phonon as well as the GaAs-like LO phonon confined in the $\mathrm{In}_{0.53} \mathrm{Ga}_{0.47} \mathrm{As}$ wells.

A comparison to the Gaussian fits reveals an asymmetry of the phonon amplitudes as a function of the $\mathrm{BO}$ frequency giving new insight into coupled Bloch-phonon dynamics in narrow-well superlattices. The observed asymmetry is in stark contrast to previous studies in $\mathrm{GaAs} / \mathrm{Al}_{0.3} \mathrm{Ga}_{0.7} \mathrm{As}$ superlattices, ${ }^{11}$ where the coherent phonon amplitude shows a sharp drop above the phonon frequency due to a resonantly increased BO dephasing, i.e., a resonantly decreased driving force of the coherent phonons. ${ }^{10} \mathrm{We}$ attribute the amplitude asymmetry observed in our measurements to the rapid $\mathrm{BO}$ dephasing that leads to an additional driving force of coherent phonons arising from tunneling processes of the optically excited electrons. ${ }^{8}$ When the tunneling times become faster than half a $\mathrm{BO}$ period the dephasing of $\mathrm{BO}$ is governed by the field dependence of the tunneling time and not any more by the dephasing due to phonon emission. However, this would lead to reduced driving force for coherent LO phonons, if the the polarization associated with the tunneling into the continuum states would not itself provide a large enough bandwidth for coherent LO phonon excitation. From the asymmetry of the phonon amplitude, i.e., higher amplitude at higher electric field, we conclude that the tunneling into continuum states acts as a driving force of coherent phonons. This excitation mechanism superimposed on the resonant Bloch-phonon coupling leads to an asymmetric increase of the coherent phonon amplitudes above the LO phonon frequencies compared to the amplitudes below the phonon resonances.

The peak position of the $\mathrm{LO}_{\mathrm{III}}$ phonon at a $\mathrm{BO}$ frequency of $10.1 \mathrm{THz}$ is shifted below the corresponding eigenfrequency by $0.9 \mathrm{THz}$. Additionally, the asymmetry observed for the two low-frequency phonons is not present for the $\mathrm{LO}_{\text {III }}$ within the frequency range investigated. Since the LO ${ }_{\text {III }}$ phonon is confined in the $\operatorname{In}_{0.52} \mathrm{Al}_{0.48} \mathrm{As}$ barriers of the superlattice, the coupling between $\mathrm{BO}$ and optical phonons of the barrier material is observed. The redshift of the amplitude peak position is attributed to two effects. (i) The "intrinsic" BO amplitude-neglecting the delocalization of electronic states due to tunneling ${ }^{8}$ - is strongly reduced due to the WS localization at the highest electric fields which corresponds to a decreased driving force of the coherent phonons, although $\mathrm{BO}$ are in resonance with the phonon frequency. (ii) The tunneling rate may be too slow to provide a large enough bandwidth for the coherent excitation of the $\mathrm{LO}_{\text {III }}$ phonon with a frequency of $11 \mathrm{THz}$.

In the following, we focus on the coupling mechanism between the electronic dynamics and the optical phonons in the superlattice. Continuous wave Raman experiments show that doubly resonant Raman scattering leads to a resonant amplitude enhancement of optical phonons in a superlattice, when the energy splitting between adjacent WS states equals the phonon energy. ${ }^{18}$ This situation is similar to the excitation of $\mathrm{BO}$ in a time-resolved experiment, where the $\mathrm{BO}$ frequency equals the optical phonon frequency. In addition, impulsively stimulated Raman scattering (ISRS) has been shown to be the driving mechanism for coherent LO phonons in GaAs/AlGaAs multiple quantum wells at low excitation densities. ${ }^{19}$ Therefore, ISRS may also contribute to the resonant enhancement of the phonon amplitudes in our experiments. ISRS should obey a strong phonon amplitude dependence on the polarization of the pump pulse due to the symmetry of the Raman tensor.

To clarify the influence of ISRS on the coherent phonon excitation in biased superlattices, REOS measurements were performed at a reverse bias of $-5.6 \mathrm{~V}$, i.e., in resonance of 


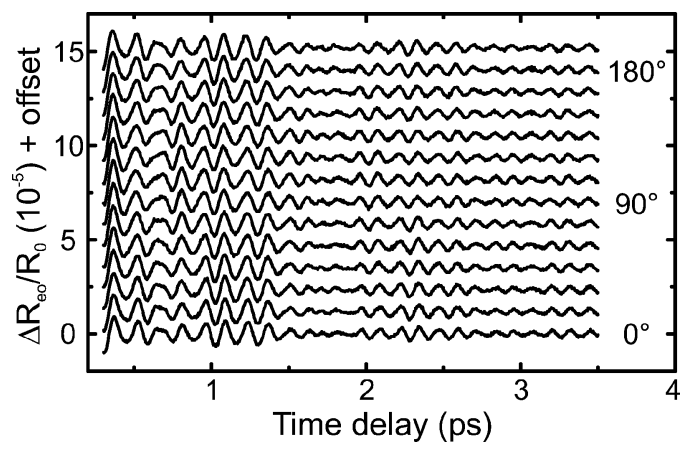

FIG. 4. Extracted REOS oscillations for different pump beam polarizations detected at a reverse bias of $-5.6 \mathrm{~V}$, i.e., under resonance condition for the $\mathrm{LO}_{\text {II }}$ phonon. The polarization angle is measured with respect to the [110] direction of the superlattice.

$\mathrm{BO}$ to the $\mathrm{LO}_{\mathrm{II}}$ phonon, under rotation of the pump beam polarization. The oscillatory contributions to these data are depicted in Fig. 4, where the pump beam polarization is measured with respect to the [110] direction of the superlattice. Obviously, the phase and the amplitudes of the coherent phonon oscillations do not depend on the pump beam polarization. For a dominant contribution of ISRS to the coherent phonon excitation, the oscillations detected at polarizations along the [110] and the $[1 \overline{1} 0]$ directions would exhibit a phase shift of about $\pi$ while the phonon amplitudes detected at polarizations along the [100] and the [010] directions would be strongly reduced. Since these effects are not observed here, the polarization dependent measurements clearly show that the coherent phonon excitation is dominated by Coulomb coupling to the carrier dynamics and that ISRS can be neglected.

In conclusion, we presented an experimental study of Bloch-phonon coupling in a narrow-well superlattice. The coherent amplitudes of different optical phonons are selectively enhanced when $\mathrm{BO}$ are tuned into resonance with the lattice frequencies. The coupling of BO to optical phonons confined in the barriers has been observed. A tunnelinginduced BO dephasing in the shallow bound miniband leads to an additional excitation process of coherent phonons at high electric fields. Here, the coherent phonons serve as a probe of tunneling processes into the above-barrier continuum. Polarization dependent measurements exclude contributions of impulsive Raman scattering to the coherent phonon excitation.

The authors thank A. Bartels for helpful discussions. The Deutsche Forschungsgemeinschaft, the Ministerium für Schule, Wissenschaft und Forschung Nordrhein-Westfalen, and the European Research Office of the U.S. Army are gratefully acknowledged for financial support.
*Electronic address: foerst@iht-ii.rwth-aachen.de

${ }^{1}$ K. Leo, Semicond. Sci. Technol. 13, 249 (1998).

${ }^{2}$ F. Bloch, Z. Phys. 52, 555 (1928).

${ }^{3}$ C. Zener, Proc. R. Soc. London, Ser. A 145, 523 (1934).

${ }^{4}$ J. Feldmann, K. Leo, J. Shah, D. A. B. Miller, J. E. Cunningham, T. Meier, G. von Plessen, A. Schulze, P. Thomas, and S. Schmitt-Rink, Phys. Rev. B 46, 7252 (1992).

${ }^{5}$ K. Leo, P. H. Bolivar, F. Brüggemann, R. Schwedler, and K. Köhler, Solid State Commun. 84, 943 (1992).

${ }^{6}$ A. Sibille, J. F. Palmier, and F. Laruelle, Phys. Rev. Lett. 80, 4506 (1998).

${ }^{7}$ M. Helm, W. Hilber, G. Strasser, R. D. Meester, F. M. Peeters, and A. Wacker, Phys. Rev. Lett. 82, 3120 (1999).

${ }^{8}$ B. Rosam, D. Meinhold, F. Löser, V. G. Lyssenko, S. Glutsch, F. Bechstedt, F. Rossi, K. Köhler, and K. Leo, Phys. Rev. Lett. 88, 1307 (2001).

${ }^{9}$ J. Hader, T. Meier, S. W. Koch, F. Rossi, and N. Lindner, Phys. Rev. B 55, 13799 (1997).

${ }^{10}$ A. W. Ghosh, L. Jönsson, and J. W. Wilkins, Phys. Rev. Lett. 85, 1084 (2000).

${ }^{11}$ T. Dekorsy, A. Bartels, H. Kurz, K. Köhler, R. Hey, and K. Ploog, Phys. Rev. Lett. 85, 1080 (2000).

${ }^{12}$ Properties of Lattice-Matched and Strained InGaAs, edited by P.
Bhattacharya (INSPEC, London, 1993).

${ }^{13}$ L. Pavesi, R. Houdré, and P. Gianozzi, J. Appl. Phys. 78, 470 (1999).

${ }^{14}$ T. Dekorsy, P. Leisching, K. Köhler, and H. Kurz, Phys. Rev. B 50, 8106 (1994).

${ }^{15}$ At higher voltages, the overlap of the broadband Bloch peaks with the sharp phonon lines inhibits an unambiguous determination of the peak positions. Note that the laser bandwidth of 50 $\mathrm{meV}$ allows the optical excitation of Bloch oscillations with frequencies above $12 \mathrm{THz}$.

${ }^{16}$ It is well known that inhomogenous electric fields in superlattices embedded in Schottky diodes result in an increase of the BO dephasing at higher electric fields. Since we investigate the carrier dynamics in a $p-i-n$ diode, such field inhomogenities can be excluded to contribute to the rapid dephasing.

${ }^{17}$ T. Dekorsy, G. C. Cho, and H. Kurz, in Light Scattering in Solids VIII, edited by M. Cardona and G. Güntherodt (Springer Verlag, Berlin, 2000), p. 169.

${ }^{18}$ F. Agulló-Rueda, E. E. Mendez, and J. M. Hong, Phys. Rev. B 38, 12720 (1988).

${ }^{19}$ K. J. Yee, Y. S. Lim, T. Dekorsy, and D. S. Kim, Phys. Rev. Lett. 86, 1630 (2001). 